\title{
Efficient harmony search algorithm for multi-stages scheduling problem for power systems degradation
}

\author{
A. Zeblah · S. Hadjeri · E. Chatelet · Y. Massim
}

Received: 8 April 2009 / Accepted: 13 June 2010 / Published online: 7 July 2010

(C) The Author(s) 2010. This article is published with open access at Springerlink.com

\begin{abstract}
Usually power energy demand increases randomly with time. To enhance system performance, expansion-planning to adapt the power system capacity to the demand is predicted. This paper uses a harmony search metaheuristic optimization method to solve the multi-stage expansion problem for multi-state series-parallel power systems. The study horizon is divided into several periods. At each period the demand distribution is forecasted in the form of a cumulative demand curve. A multiple-choice of additional components from a list of available products can be chosen and included into any subsystem component at any stage to improve the system performance. The components are characterized by their cost, performance (capacity), and availability. The objective is to minimize each investment over its study period while satisfying availability or performance constraints. A universal generating function technique is applied to evaluate power system availability. The harmony search approach is required to identify the optimal combination of adding components with different parameters to be allocated in parallel at each stage.
\end{abstract}

\author{
A. Zeblah $(\varangle) \cdot$ S. Hadjeri \\ Engineering Faculty, University of Sidi Bel Abbes, Sidi Jilali, \\ 22000 Sidi Bel Abbes, Algeria \\ e-mail: azeblah@yahoo.fr \\ S. Hadjeri \\ e-mail: Shadjeri2@yahoo.fr \\ E. Chatelet \\ Institut Charles Delaunay (CNRS FRE 2848), LM2S, \\ University of Technology of Troyes, 12 rue Marie Curie, \\ Troyes Cedex, France \\ e-mail: Eric.chatelet@utt.fr \\ Y. Massim \\ Faculty of Science, University of Sidi Bel Abbes, Gumbeta, \\ BP 89, 22000 Sidi Bel Abbes, Algeria \\ e-mail: yamanimassim@yahoo.fr
}

Keywords Expansion-planning · Harmony search · Redundancy optimization · Power system · Universal generating moment function

\section{Introduction}

In many industrial systems, expansion planning system is considered as an important problem in design, e.g., in power systems, water distribution and in manufacturing systems. Such as modifying existing structure, designing a new structure and adding $r(k)^{*}$ or retrieving $r(k)^{* *}$ components belonging to the redundancy optimization problem as suggested in [1]. This latter is a well known combinatorial optimization problem where adding components is achieved by numerous discrete choice made from components available on the market. Based on the cost, availability and performance, the objective function is to minimize the investmentcosts over each study period within the planning horizon for a certain availability or (reliability) requirement. Figure 1 shows the typical series-parallel power structure. However, the capacity of many production systems is defined by multiple, heterogeneous units. In this situation the system can have a several-range level of performance depending from perfect working to total failure; in this case it is considered as a multi-state system (MSS).

The MSS system consists of $n$ subsystems $C_{i}(i=$ $1,2, \ldots, n)$ in series arrangement. Each subsystem $C_{i}$ can contain several components of type $i$ connected in parallel from various versions, which are proposed by the suppliers on the market. Each version in turn can contain one or more identical components in parallel. Components are characterized by their cost, availability and performance according to their version. Different versions of components may be chosen for any given subsystem component. Thus, our study is 


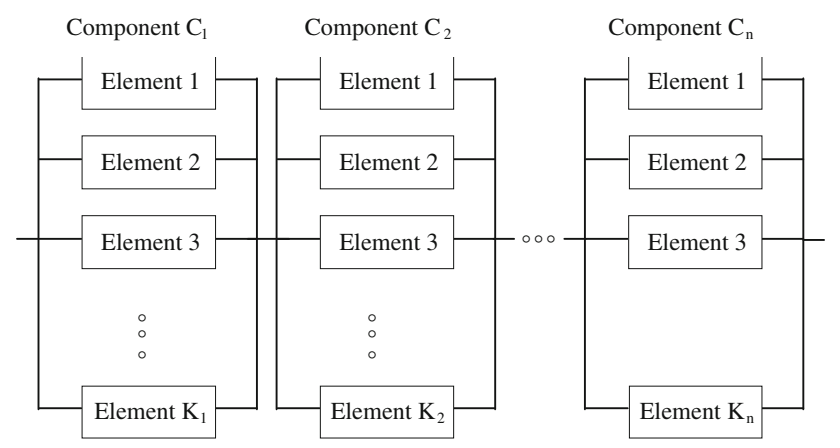

Fig. 1 Series-parallel power system structure

not limited to the case where only the homogeneous components are used. Beside a lot of alternatives lead to change the performance and reliability as in series-parallel replacement and modernization problems [2,3]. The simplest method in this work to help system performance to face to the increase demand is its expansion.

\subsection{Literature review}

The classical reliability theory is based on the binary assumption that the system is either working perfectly or completely failed. However, in many real life situations we are actually able to distinguish among various levels of performance for both subsystem and its components. In this case, it is important to develop MSS reliability theory. Most of research works in MSS reliability (availability) analysis extend the results in binary state system theory to the multi-state case. A good and extensive recent review of the literature can be found for example in [4] or [5]. Generally, the methods of MSS reliability assessment are based on many different approaches such as: the structure function approach, the stochastic process (mainly Markov) approach, the simulation technique (Monte-Carlo), and the universal moment generating function (UMGF) approach.

The problem of total investment-cost minimization, subject to reliability or availability constraints, is well known as the redundancy optimization problem (ROP). The ROP for series parallel systems is NP-hard [6] and has been studied in many different forms as summarized in [7] and more recently in [8]. In most existing works on ROP, it is usually assumed that a system may experience only two possible states. The ROP for the multi-state reliability was introduced in [9]. In [10] and [1], genetic algorithms were used to find the optimal or nearly optimal power system structure. In [11] the multi-stage expansion-planning problem for multi-state series-parallel system is solved by using a genetic algorithm as an optimization tool.

This paper uses a harmony search optimization to solve the multi-stage expansion-planning problem for multi-state series-parallel electrical power system. The idea of employing a harmony search to solve combinatorial optimization problems was recently proposed in [12]. It has been recently adapted for the reliability design of binary state systems [13]. The harmony search method has not yet been used for the multi-stages expansion problem.

\subsection{Approach and outline}

As the problem formulated in this paper is a complicated combinatorial optimization one, an exhaustive examination of all possible solutions is not realistic considering reasonable time limitations. Because of this, the harmony search optimization (HSO) will be adapted to the multi-stage expansionplanning problem to find optimal or nearly optimal solutions to be obtained in a short time. The harmony search is inspired by the behavior of real musicians based on natural musical performance processes that occur when a musician searches for a better state of harmony, such as during jazz improvisation. Jazz improvisation seeks to find musically pleasing harmony (a perfect state) as determined by an aesthetic standard, just as the optimization process seeks to find a global solution (a perfect state) as determined by an objective function. The pitch of each musical instrument determines the aesthetic quality, just as the objective function value is determined by the set of values assigned to each design variable [14].

To evaluate the availability of a given selected structure of the series-parallel system, a fast procedure of availability estimation is developed. This procedure is based on a modern mathematical technique: the $z$-transform or universal moment generating function which was introduced in [15]. It was proven to be very effective for high-dimension combinatorial problems: see e.g. [4,5] and references therein. The universal moment generating function is an extension of the ordinary moment generating function. The method developed in this paper allows the availability function of reparable series-parallel MSS to be obtained using a straightforward numerical procedure.

The remainder of this paper is outlined as follows: in Sect. 2, the expansion-planning problem EPP is formulated. In Sect. 3, availability evaluation method is developed. In Sect. 4, the harmony search optimization method is adapted to solve the expansion-planning problem. In Sect. 5, illustrative examples and numerical results are presented in which the optimal choice of components in a subsystem is found. Conclusions are drawn in Sect. 6.

\section{Expansion-planning problem}

There has been much interest in production scheduling models, where expansion-planning (EPP) is considered. To 


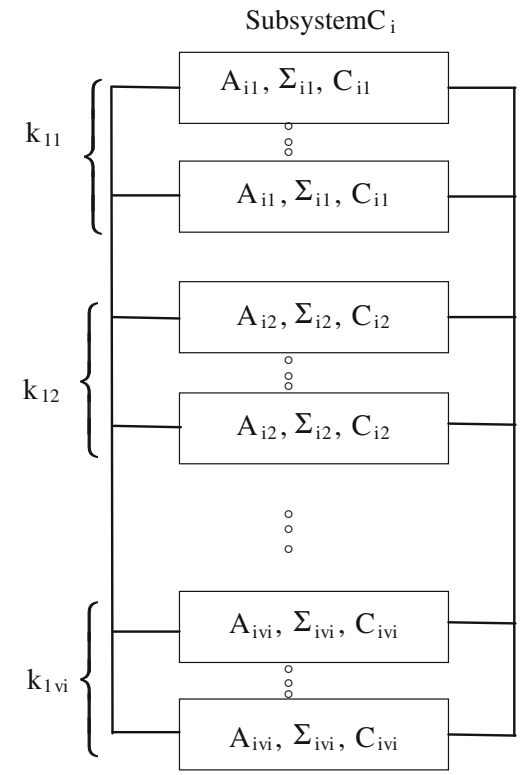

Fig. 2 Detailed structure of a given subsystem

formulate the expansion-planning problem of power system, let us consider a series-parallel power system containing $n$ subsystems $C_{i}(i=1,2, \ldots, n)$ in series arrangement as sketched in Fig. 1. Each component $C_{i}$ in turn contains a number of different components connected in parallel. All components of any given subsystem component belonging to different version $v$. Components are characterized by their availability $\left(A_{i v}\right)$, cost $\left(C_{i v}\right)$ and performance $\left(\Sigma_{i v}\right)$ according to their version. The structure of subsystem component $i$ can be defined by the numbers of parallel components (of each version) $k_{i v}$ for $1 \leq v \leq V_{i}$, where $V_{i}$ is a number of versions available for component of type $i$. Figure 2 illustrates these notations for a given component $i$. Each version $k_{i v}$ contains $m$-identical components which are also connected in parallel. The entire system can therefore be defined by the set of triplets $\boldsymbol{k}_{\boldsymbol{0}}=$ $\left\{A_{i v}, \sum_{i v}, C_{i v}\right\}\left(1 \leq i \leq n, 1 \leq v \leq V_{i}\right)$. Where $\boldsymbol{k}_{\boldsymbol{0}}$ : represent the initial system structure. In fact, for given triplet $\boldsymbol{k}_{\boldsymbol{0}}$, the total cost of the initial power system structure can be calculated as:

$C_{0}=\sum_{i=1}^{n} \sum_{v=1}^{V_{i}} k_{i v} C_{i v}$

\subsection{Partial optimal design problem}

The multi-state EPP of electrical power system at the initial period can be formulated as follows: find the minimal cost system configuration $\boldsymbol{k}_{1}, \boldsymbol{k}_{2}, \ldots, \boldsymbol{k}_{\boldsymbol{n}}$, such that the corresponding availability exceeds or equal the specified avail- ability $A_{0}$. That is,

Minimize $\quad C_{0}=\sum_{i=1}^{n} \sum_{v=1}^{V_{i}} k_{i v} C_{i v}$

subject to $\quad A\left(\boldsymbol{k}_{\boldsymbol{l}}, \boldsymbol{k}_{2}, \ldots, \boldsymbol{k}_{\boldsymbol{n}}, \boldsymbol{D}, \boldsymbol{T}\right) \geq A_{0}$

\subsection{Determination of the expansion plan}

In the cases when the production system is not able to satisfy the consumer demand. The existing system structure must be reinforced. This is way to enhance system performance and/or reliability to be improved. This solution is more realistic, practical and rather than designing a new structure. Indeed, the system should be expanded at different stage of the study period $Y$, in which the load curve demand varies from stage to stage. Each stage $\theta$ begins $\tau(\theta)$ years after the initial stage (i.e., initial stage $\theta$ ). To provide the desired level of productivity and reliability, systematically, the addition or retrieval by replacement of components can be chosen from different versions of any given subsystem component. To distinguish between the components' characteristics of the existing structure and the adding components the notation of component versions is introduced. For each subsystem component $i$ there are several component versions available in the market. A set of parameters contain the nominal capacity $\left(\Sigma_{i h}\right)$, availability $\left(A_{i h}\right)$ and cost $\left(C_{i h}\right)$ is specified for each version $h$ of component of type $i$. Therefore, component can be defined by $r_{i h}(\theta)\left(1 \leq h \leq H_{l}\right)$ where $H_{l}$ define the total number versions of the expanding components.

The entire expansion system may be defined by the vectors $\boldsymbol{r}_{i}(\theta)=\left\{r_{i h}\right\}\left(1 \leq i \leq n, 1 \leq h \leq H_{l}\right)$ at stage $(\theta)$. For a given set of $\boldsymbol{r}_{1}(\theta), \boldsymbol{r}_{2}(\theta), \ldots, \boldsymbol{r}_{n}(\theta)$, the total cost of the system expansion at stage $(\theta)$ can be calculated at the present value as:

$C(\theta)=\sum_{i=1}^{n} \sum_{h=1}^{H_{l}} r_{i h}(\theta) C_{i h} \pm \frac{1}{(1+\mathrm{IP})^{\tau(\theta)}} C_{0}$

where IP represents the interest rate.

For given expansion structure $\boldsymbol{k}$ defined by the vectors $\boldsymbol{k}=\left\{\boldsymbol{r}_{\boldsymbol{I}}(\theta), \boldsymbol{r}_{\boldsymbol{2}}(\theta), \ldots, \boldsymbol{r}_{\boldsymbol{n}}(\theta)\right\}$, the total cost investment during all the study period $Y$ can be calculated as follows

$C(k)=\sum_{\theta=1}^{Y}\left(\sum_{i=1}^{n} \sum_{h=1}^{H_{l}} r_{i h}(\theta) C_{i h} \pm \frac{1}{(1+\mathrm{IP})^{\tau(\theta)}} C_{0}\right)$

\subsection{Total optimal design problem}

The multi-state system redundancy expansion optimization can be formulated as follows: find the minimal cost of system expansion structure $\left.\boldsymbol{k}_{0}, \boldsymbol{k}, \boldsymbol{D}(\theta), \boldsymbol{T}(\theta)\right)$ that meets or exceeds 
the required availability $A_{0}$. That is,

$$
\begin{aligned}
\operatorname{Minimize} \quad C(k)= & \sum_{\theta=1}^{Y}\left(\sum_{i=1}^{n} \sum_{h=1}^{H_{l}} r_{i h}(\theta) C_{i h}\right. \\
& \left. \pm \frac{1}{(1+\mathrm{IP})^{\tau(\theta)}} \sum_{i=1}^{n} \sum_{v=1}^{V_{i}} k_{i v} C_{i v}\right)
\end{aligned}
$$

Subject to $A\left(\boldsymbol{k}_{\boldsymbol{0}}, \boldsymbol{k}, \boldsymbol{D}(\theta), \boldsymbol{T}(\theta)\right) \geq A_{0}$

To estimate the availability index at each stage of study, it is necessary to calculate the overall probability that the load demand corresponding to this stage is not meet. This method is referred to the availability of reparable multi-state system that is developed in the next section.

\subsection{Availability of reparable multi-state systems}

The considered system is a series-parallel power system composed of a number of failure prone units, such that the failure of some components leads only to a degradation of the system performance. This system is considered to have a range of performance levels from perfect functioning to complete failure. In fact, the system failure can lead to decreased capability to accomplish a given task, but not to complete failure. An important MSS measure is related to the ability of the system to satisfy a given demand.

When applied to electric power systems, reliability is considered as a measure of the ability of the system to meet the load demand $(D)$, i.e., to provide an adequate supply of electrical energy $(\Sigma)$. This definition of the reliability index is widely used for power systems: see e.g., [16-18] and [1]. The loss of load probability (LOLP) index is usually used to estimate the reliability index [19]. This index is the overall probability that the load demand will not be met. Thus, we can write $R=\operatorname{Prob}(\Sigma \geq D)$ or $R=1-$ LOLP with LOLP $=\operatorname{Probab}(\Sigma<D)$. This reliability index depends on consumer demand $D$.

For reparable MSS, a multi-state steady-state availability $E$ is used as $\operatorname{Prob}(\Sigma \geq D)$ after enough time has passed for this probability to become constant [18]. In the steady-state the distribution of states probabilities is given by Eq. (8), while the multi-state stationary availability is formulated by Eq. (9):

$P_{j}=\lim _{t \rightarrow \infty}\left[\operatorname{Prob}\left(\Sigma(t)=\Sigma_{j}\right)\right]$

$E=\sum_{\Sigma_{j} \geq D} P_{j}$

At each operation period of stage $(\theta)$ the demand distribution represented by the cumulative curve is predicted. If the period of stage $(\theta)$ is divided into $M_{\theta}$ intervals with duration's $T_{j}(\theta)\left(1 \leq j \leq M_{\theta}\right)$ and each interval has a required demand level $D_{j}(\theta)$. We denote by $\boldsymbol{D}(\theta)$ and $\boldsymbol{T}(\theta)$ the vectors $\left\{D_{j}(\theta)\right\}$ and $\left\{T_{j}(\theta)\right\}\left(1 \leq j \leq M_{\theta}\right)$, respectively. Some assumptions are taken into account, the intervals for the stages are equal and the redundant intervals $T_{j}(\theta)=0(M \leq \theta \leq Y)$. Then the generalized MSS availability index $A$ at each stage can be calculated as:

$$
A=\frac{1}{\sum_{j=1}^{M} T_{j}(\theta)} \sum_{j=1}^{M} \operatorname{Prob}\left(\Sigma_{T}(\theta) \geq D_{j}\right) T_{j}(\theta)
$$

where $\Sigma_{T}(\theta)$ define the production of system containing the initial structure and the additional components presented at stage $(\theta)$. As the availability $A$ is a function of $\boldsymbol{k}_{0}, \boldsymbol{k}, \boldsymbol{D}(\theta)$ and $\boldsymbol{T}(\theta)$, it will be written $A\left(\boldsymbol{k}_{0}, \boldsymbol{k}, \boldsymbol{D}(\theta), \boldsymbol{T}(\theta)\right)$. In the case of a power system, the vectors $\boldsymbol{D}$ and $\boldsymbol{T}$ define the cumulative load curve (consumer demand). In general, this curve is known for every power system.

\section{Multi-state system availability estimation based on UMGF method}

The procedure used in this paper is based on the universal $z$-transform, which is a modern mathematical technique introduced in [15]. This method, convenient for numerical implementation, is proved to be very effective for highdimension combinatorial problems. In the literature, the universal $z$-transform is also called universal moment generating function (UMGF) or simply $u$-function or $u$-transform. In this paper, we mainly use the acronym UMGF. The UMGF extends the widely known ordinary moment generating function.

\subsection{Definition}

The UMGF of a discrete random variable $\sum$ is defined as a polynomial:

$u(z)=\sum_{j=1}^{J} P_{j} z^{\Sigma_{j}}$

where the variable $\Sigma$ has $J$ possible values and $P_{j}$ is the probability that $\Sigma$ is equal to $\Sigma_{j}$.

The probabilistic characteristics of the random variable $\Sigma$ can be found using the function $u(z)$. In particular, if the discrete random variable $\Sigma$ is the MSS stationary output performance, the availability $E$ is given by the probability $\operatorname{Prob}(\Sigma \geq D)$ which can be defined as follows:

$\operatorname{Probab}(\Sigma \geq D)=\Psi\left(u(z) z^{-D}\right)$ 
where $\Psi$ is a distributive operator defined by expressions (13) and (14):

$$
\begin{aligned}
& \Psi\left(P z^{\sigma-D}\right)= \begin{cases}P, & \text { if } \sigma \geq D \\
0, & \text { if } \sigma<D\end{cases} \\
& \Psi\left(\sum_{j=1}^{J} P_{j} z^{\Sigma_{j}-D}\right)=\sum_{j=1}^{J} \Psi\left(P_{j} z^{\Sigma_{j}-D}\right)
\end{aligned}
$$

It can be easily shown that Eqs. (13) and (14) meet condition $\operatorname{Prob}(\Sigma \geq D)=\sum_{\Sigma_{j} \geq D} P_{j}$. By using the operator $\Psi$, the coefficients of polynomial $u(z)$ are summed for every term with $\Sigma_{j} \geq D$, and the probability that $\Sigma$ is not less than some arbitrary value $D$ is systematically obtained.

Consider single components with total failures and each component $i$ has nominal performance $\Sigma_{i}$ and availability $A_{i}$. Then, $\operatorname{Prob}\left(\Sigma=\Sigma_{i}\right)=A_{i}$ and $\operatorname{Probab}(\Sigma=\sim)=1-A_{i}$. The UMGF of such a component has only two terms and can be defined as:

$u_{i}(z)=\left(1-A_{i}\right) z^{0}+A_{i} z^{\Sigma_{i}}=\left(1-A_{i}\right)+A_{i} z^{\Sigma_{i}}$

To evaluate the MSS availability of a series-parallel system, two basic composition operators are introduced. These operators determine the polynomial $u(z)$ for a group of components.

\subsection{Parallel components}

Let us consider a system component $m$ containing $J_{m}$ components connected in parallel. As the performance measure is related to the system productivity, the total performance of the parallel system is the sum of performances of all its components. In power systems engineering, the term capacity is usually used to indicate the quantitative performance measure of a component [6]. The capacity of a component can be measured as a percentage of nominal total system capacity. In power system, components are generators units, transformer and transmission lines. Therefore, the total performance of the parallel unit is the sum of performances.

The $u$-function of MSS component $m$ containing $J_{m}$ parallel components can be calculated by using the $\Gamma$ operator:

$$
\begin{gathered}
u_{p}(z)=\Gamma\left(u_{1}(z), u_{2}(z), \ldots, u_{n}(z)\right), \\
\text { where } \Gamma\left(g_{1}, g_{2}, \ldots, g_{n}\right)=\sum_{i=1}^{n} g_{i} .
\end{gathered}
$$

Therefore for a pair of components connected in parallel:

$\Gamma\left(u_{1}(z), u_{2}(z)\right)=\Gamma\left(\sum_{i=1}^{n} P_{i} z^{a_{i}}, \sum_{j=1}^{m} Q_{j} z^{b_{j}}\right)$.

Parameters $a_{i}$ and $b_{j}$ are physically interpreted as the respective performances of the two components. $n$ and $m$ are numbers of possible performance levels for these components.
$P_{i}$ and $Q_{j}$ are steady-state probabilities of possible performance levels for components. One can see that the $\Gamma$ operator is simply a product of the individual $u$-functions. Thus, the component UMGF is:

$u_{p}(z)=\prod_{j=1}^{J_{m}} u_{j}(z)$

Given the individual UMGF of components defined in Eq. (15), we have:

$u_{p}(z)=\prod_{j=1}^{J_{m}}\left(1-A_{j}+A_{j} z^{\Sigma_{i}}\right)$.

\subsection{Series components}

When the components are connected in series, the component with the least performance becomes the bottleneck of the system. This component therefore defines the total system productivity. To calculate the $u$-function for system containing $n$ components connected in series, the operator $\eta$ should be used: $u_{s}(z)=\eta\left(u_{1}(z), u_{2}(z), \ldots, u_{m}(z)\right)$, where $\eta\left(g_{1}, g_{2}, \ldots, g_{m}\right)=\min \left\{g_{1}, g_{2}, \ldots, g_{m}\right\}$ so that

$$
\begin{aligned}
\eta\left(u_{1}(z), u_{2}(z)\right) & =\eta\left(\sum_{i=1}^{n} P_{i} z^{a_{i}}, \sum_{j=1}^{m} Q_{j} z^{b_{j}}\right) \\
& =\sum_{i=1}^{n} \sum_{j=1}^{m} P_{i} Q_{j} z^{\min \left\{a_{i}, b_{j}\right\}}
\end{aligned}
$$

Applying composition operators $\Gamma$ and $\eta$ consecutively, one can obtain the UMGF of the entire series-parallel system. To do this we must first determine the individual UMGF of each component. The next section presents the harmony search meta-heuristic optimization method to solve the redundancy optimization problem for multi-state expansion-planning systems.

\section{The harmony search optimization approach}

The problem formulated in this paper is a complicated combinatorial optimization problem. The total number of different solutions to be examined is very large, even for rather small problems. An exhaustive examination of the enormous number of possible solutions is not feasible given reasonable time limitations. Thus, because of the search space size of the ROP for MSS, a new meta-heuristic is developed in this section. This meta-heuristic consists in an adaptation of the harmony search optimization method. 


\subsection{The HS principle}

Recently, [20] introduced a new approach to optimization problems derived from the study of musician, called "Harmony Search Algorithm". The HS algorithm mimics musical improvisation process where the musicians try to find a better harmony. A musician always desires to reach the best harmony, which can be obtained by numerous practices. The pitches of the instruments are adjusted after each practice. The HS will be explained in the following, similar to the work in [21]. Figure 3 demonstrates the analogy between HM and power system design. HM is the most important part of HS, which is shown in Fig. 1. Jazz improvisation is the best example for clarifying the HM. Many jazz of five music instruments consist of a guitarist, double bassist, and pianist. Each musician in the trio has different pitches: guitarist (Sol, Si, Re, Fa, Mi); double bassist (Fa, Mi, Re, La, $\mathrm{Si}$ ); pianist ( $\mathrm{Si}, \mathrm{Re}, \mathrm{Mi}, \mathrm{Do}, \mathrm{La})$. Let the guitarist randomly play Sol out of its pitches ( $\mathrm{Sol}, \mathrm{Si}, \mathrm{Re}, \mathrm{Fa}, \mathrm{Mi}$ ), double bassist $\mathrm{Si}$ out of (Fa, Mi, Re, $\mathrm{La}, \mathrm{Si}$ ) and pianist $\mathrm{Re}(\mathrm{Si}, \mathrm{Re}, \mathrm{Mi}$, Do, La). Therefore, the new harmony (Sol, Si, Re) becomes another harmony (musically G-chord). If the new harmony is better than existing worst harmony in the HM, new harmony is included in the HM and the existing worst harmony is excluded from the HM. The process is repeated until the best harmony is obtained.

We consider a power system design process to built, which consists of five different design variables: First design variable is selected from generators subsystem: $x_{1}^{1} \rightarrow$ $\left\{x_{11}^{1}, \ldots, x_{18}^{1}\right\}$, second from MT/HT transformers subsystem $x_{2}^{1} \rightarrow\left\{x_{21}^{1}, \ldots, x_{25}^{1}\right\}$, third from THT lines subsystem $x_{3}^{1} \rightarrow\left\{x_{31}^{1}, \ldots, x_{34}^{1}\right\}$, fourth from HT/MT transformers subsystem $x_{4}^{1} \rightarrow\left\{x_{41}^{1}, \ldots, x_{45}^{1}\right\}$, and the end from the MT lines subsystem $x_{5}^{1} \rightarrow\left\{x_{51}^{1}, \ldots, x_{55}^{1}\right\}$. At initial stage a vector solution is selected randomly from each subsystem one and /or more components are selected. For this stage the process starting to optimize the power designs.

If the new selected power design is better than the existing worst power design, the worst power design present the highest objective function value, the new design is included, and

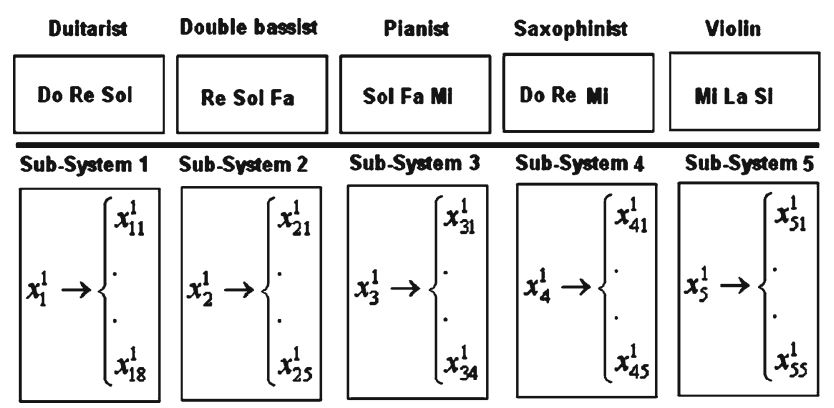

Fig. 3 Analogy between HS and power system design the worst design is excluded from vector solution of power design process. This procedure is repeated until the terminating criterion is satisfied.

An analogy is presented between the music improvisation process [20] and the optimum electrical power design can be established in the following way: The harmony denotes the power design vector; whereas the different harmonies during the improvisation represent the different power design vectors throughout the optimum power design process. Each musical instrument denotes the subsystem power design variables (set of electrical components) of objective function. The pitches of the instruments represent the design variable's values (components technology with no position). A better harmony represents local optimum, and the best harmony is the global optimum.

\subsection{HS-based initial solution approach}

The optimum design algorithm developed for power design based on harmony search method treats the sequence number of the components technology in the list selected for each subsystem components as a design variable. Once a sequence number of technology is selected then the subsystem components designation and properties of this latter become a new available for the algorithm. Hence the design vector consists of the integer numbers that are the sequence number of technologies in the discrete set. Since each row of the harmony memory matrix corresponds to a candidate for power design, the components of this matrix represent the order number of components technologies. The design algorithm consists of the following steps.

The optimum power design algorithm using $\mathrm{HS}$ is sketched basically as shown in Fig. 4.

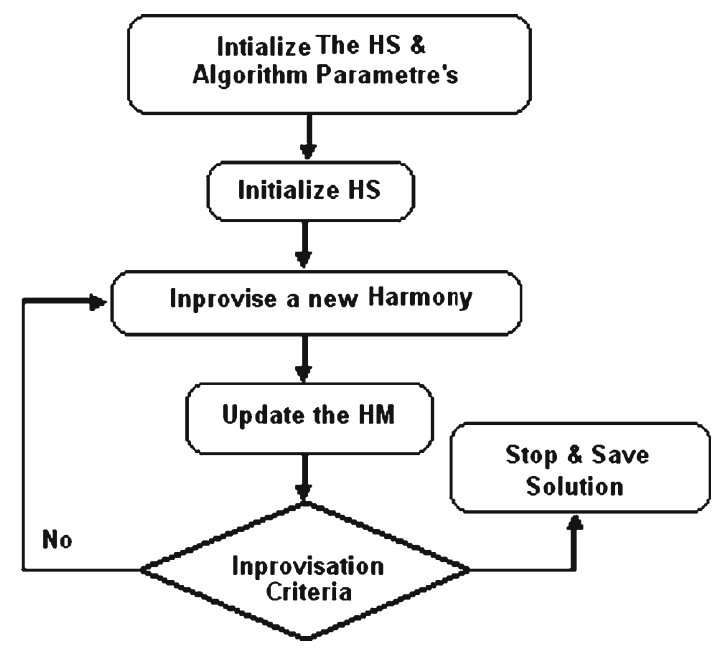

Fig. 4 Basic flowchart diagram For HS algorithm 


\subsubsection{Initialize the harmony search parameters}

The HS algorithm parameters are attained in this step. These are harmony memory size (HMS), harmony memory consideration rate (HMCR), pitch adjustment ratio (PAR), and stopping criteria (number of improvisation). These parameters are selected depending on the problem.

\subsubsection{Initialize harmony memory}

The HM matrix is filled with randomly generated designs as the HMS.

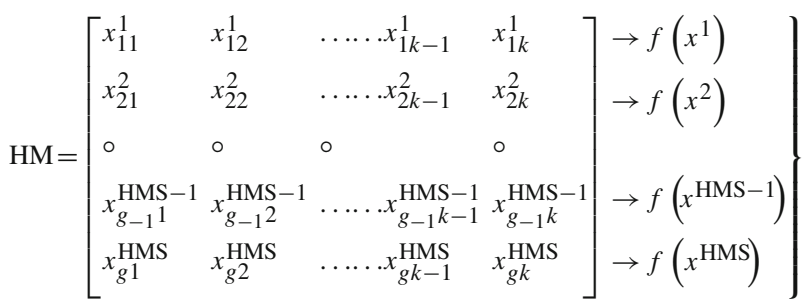

Each vector denotes a power design vectors solutions in the $\mathrm{HM} x_{1 k}^{1}, x_{2 k}^{2}, \ldots, x_{g k}^{\mathrm{HMS}}$ and the corresponding unconstrained objective function value, respectively $f\left(x^{1}\right), \ldots, f\left(x^{\mathrm{HMS}}\right)$. The power system designs are stored by the unconstrained objective function values $f\left(x^{1}\right) \prec f\left(x^{2}\right), \ldots, \prec f\left(x^{\mathrm{HMS}}\right)$. The aim of using HM is to preserve better power systems designs in the search process.

Calculate the initial cost power design at the initial stage: $C_{0}$ Calculate the availability of the initial power design at the initial stage: $A_{0}$.

\subsubsection{Improvise a new harmony}

A new harmony $\left[x^{n v}\right]=\left\{x_{11}^{n v}, x_{12}^{n v}, \ldots, x_{g k}^{n v}\right\}$ is improvised from either the HM or entire technologies from each subsystem's list. Three rules are used for the generation of a new harmony. These are HM consideration, pitch adjustment, and random generation. In the $\mathrm{HM}$ consideration, the value of the first power design variable $\left[x_{g_{-1}, 1}^{n v}\right]$ for a new harmony is chosen from any value HM (i.e. $\left\{x_{1 k}^{1}, \ldots, x_{g k}^{\mathrm{HMS}}\right\}$ ) or entire available technology in the market denoted the available technologies by $\left[Y_{A T}\right]^{2}$. HMCR is applied as follows:

$$
\begin{cases}x_{g i}^{n v} \in\left\{x_{g i}^{1}, x_{g i}^{2}, \ldots, x_{g i}^{\mathrm{HMS}}\right\} & \text { if ran } \leq \mathrm{HMCR} \\ x_{g i}^{n v} \in Y_{T A} & \text { if ran } \succ \text { HMCR }\end{cases}
$$

(ran) is the random number uniformly distributed over the interval $[0,1]$ and generated. If the ran number is less than the HMCR value, $i$ th power design variable of new power design $\left[x_{g k}^{n v}\right]$ is selected from the current values stored in the $i$ th column of HM. Otherwise the $i$ th power design variable of new power design $\left[x_{g k}^{n v}\right]$ is selected from the entire available technologies list $\left[Y_{A T}\right]$.

Any power system design variable of the new harmony, $\left[x^{n v}\right]=\left\{x_{11}^{n v}, x_{12}^{n v}, \ldots, x_{g k}^{n v}\right\}$, obtained by the memory consideration is examined to determine whether it is pitchadjusted or not. Pitch adjustment is made by PAR. PAR investigates better power system design in the neighboring of the current power system design. PAR is applied as follows:

PAD for $x_{g i}^{n v} \begin{cases}\text { Yes } & \text { if } \operatorname{ran}_{1} \leq \mathrm{PAR} \\ \mathrm{No} & \text { if } \operatorname{ran}_{1} \succ \mathrm{PAR}\end{cases}$

$\left(\operatorname{ran}_{1}\right)$ is the random number uniformly distributed over the interval $[0,1]$ and generated. If this random number is less than the PAR, $x_{g i}^{n v}$ is replaced with its neighbor technology components list. If this random number is not less than PAR, $x_{g i}^{n v}$ remains the same.

\subsubsection{Update the harmony memory}

If the new harmony $\left[x^{n v}\right]=\left\{x_{11}^{n v}, x_{12}^{n v}, \ldots, x_{g k}^{n v}\right\}$ is better than the worst power design in the HM, the new power design is included in the HM, and the existing worst harmony is excluded from the HM. In this process, it should be noted that HM matrix is sorted again by unconstrained objective function and the same power design is not permitted in the HM more than once.

\subsubsection{Stopping criterion}

The steps in Sects. 4.2.3 and 4.2.4 are repeated until a termination criterion is satisfied or reaching the number of improvisation (NI).

Calculate the initial optimal cost power design at the initial stage: $\tau(0)=0 \rightarrow C_{0}$

Calculate the availability of the optimal power design at the initial stage: $\tau(0)=0 \rightarrow A$.

Considering now the case when the power demand increases or decreases randomly. For any stage $\tau(\theta) \neq 0$, the process starts by proposing the following method.

\subsection{HS-based solution approach for EPP}

The design algorithm of EPP consists of the following steps. The optimum power design algorithm using HS is sketched basically as shown in Fig. 5 . 


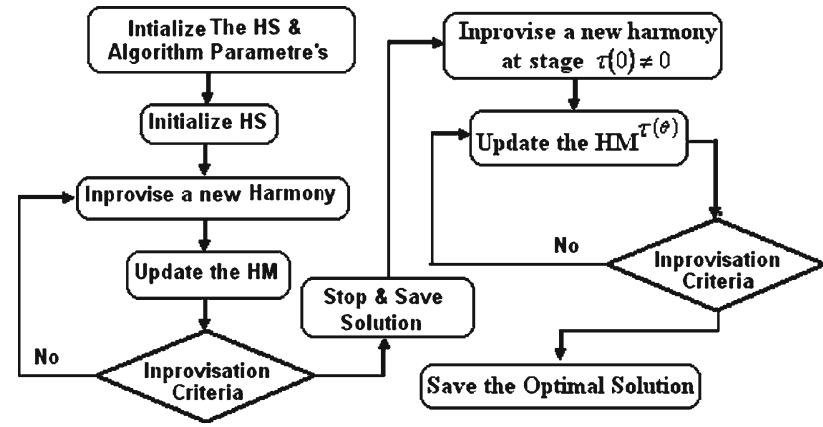

Fig. 5 Basic flowchart diagram Of HS algorithm For EPP

\subsubsection{Using the filled harmony memory}

The HM matrix is filled with the first optimal solution at the initial stage $\tau(0)=0$.

$$
\begin{aligned}
& \mathrm{HM}^{\tau(0)} \\
& \left.\quad=\left[\begin{array}{lllll}
x_{11}^{\tau(0) 1} & x_{12}^{\tau(0) 1} & \ldots & x_{1 k-1}^{\tau(0) 1} & x_{1 k}^{\tau(0) 1}
\end{array}\right] \rightarrow f\left(x^{\tau(0) 1}\right)\right\}
\end{aligned}
$$

Then begin to fill the $\mathrm{HM}^{\tau(\theta)}$ matrix with randomly generated designs as the HMS ${ }^{\tau(\theta)}$ for the next stage $\tau(0) \neq 0$.

$$
\begin{aligned}
& \mathrm{HM}^{\tau(\theta)} \\
& =\left[\begin{array}{llll}
x_{11}^{\tau(\theta) 1} & x_{12}^{\tau(\theta) 1} & \ldots x_{1 k-1}^{\tau(\theta) 1} & x_{1 k}^{\tau(\theta) 1} \\
x_{21}^{\tau(\theta) 2} & x_{22}^{\tau(\theta) 2} & \ldots x_{2 k-1}^{\tau(\theta) 2} & x_{2 k}^{\tau(\theta) 2} \\
\bullet & \bullet & \bullet & \bullet \\
x_{g 1}^{\tau(\theta) \mathrm{HMS}} & x_{g 2}^{\tau(\theta) \mathrm{HMS}} & \ldots x_{g k-1}^{\tau(\theta) \mathrm{HMS}} & x_{g k}^{\tau(\theta) \mathrm{HMS}}
\end{array}\right] \rightarrow f\left(x^{\tau(\theta) 1}\right) \\
& \rightarrow f\left(x^{\tau(\theta) 2}\right) \\
&
\end{aligned}
$$

Each vector denotes a power design vectors solutions in the $\operatorname{HM}^{\tau(\theta)} x_{1 k}^{\tau(\theta) 1}, x_{2 k}^{\tau(\theta) 2}, \ldots, x_{g k}^{\tau(\theta) \text { HMS }}$ and the corresponding unconstrained objective function value, respectively $f\left(x^{\tau(\theta) 1}\right), \ldots, f\left(x^{\tau(\theta) \text { HMS }}\right)$. The power system designs are stored by the unconstrained objective function values $f\left(x^{\tau(\theta) 1}\right) \prec f\left(x^{\tau(\theta) 2}\right), \ldots, \prec f\left(x^{\tau(\theta) \text { HMS }}\right)$. The aim of using $\mathrm{HM}^{\tau(\theta)}$ is to preserve better power systems designs in the search process.

Calculate the initial cost power design at stage $\tau(0) \neq 0 \rightarrow$ $C(k)$

Calculate the availability of the initial power design at the stage $\tau(0) \neq 0 \rightarrow A_{0}$.

\subsubsection{Improvise a new harmony}

A new harmony $\left[x^{\tau(\theta) n v}\right]=\left\{x_{11}^{\tau(\theta) n v}, x_{12}^{\tau(\theta) n v}, \ldots, x_{g k}^{\tau(\theta) n v}\right\}$ is improvised from either the $\operatorname{HM}^{\tau(\theta)}$ or entire technologies from each subsystem's list. Three rules are used for the generation of a new harmony. These are $\mathrm{HM}^{\tau(\theta)}$ consideration, pitch adjustment, and random generation. In the
$\mathrm{HM}^{\tau(\theta)}$ consideration, the value of the first power design variable $\left[x_{g_{-1}, 1}^{\tau(\theta) n v}\right]$ for a new harmony is chosen from any value $\mathrm{HM}^{\tau(\theta)}$ (i.e. $\left\{x_{1 k}^{\tau(\theta) 1}, \ldots, x_{g k}^{\tau(\theta) \mathrm{HMS}}\right\}$ ) or entire available technology in the market denoted the available technologies by $\left[Y_{A T}^{\tau(\theta)}\right]^{2} . \mathrm{HMCR}^{\tau(\theta)}$ is applied as follows:

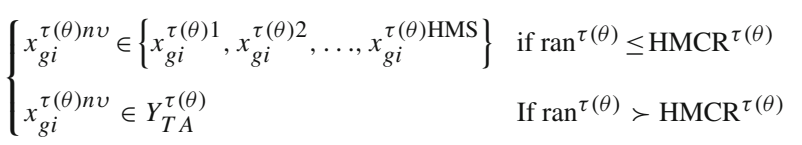

$(\operatorname{ran})^{\tau(\theta)}$ is the random number uniformly distributed over the interval $[0,1]$ and generated. If the $\operatorname{ran}^{\tau(\theta)}$ number is less than the $\operatorname{HMCR}^{\tau(\theta)}$ value, $i$ th power design variable of new power design $\left[x_{g k}^{\tau(\theta) n v}\right]$ is selected from the current values stored in the $i$ th column of $\operatorname{HM}^{\tau(\theta)}$. Otherwise the $i$ th power design variable of new power design $\left[x_{g k}^{\tau(\theta) n v}\right]$ is selected from the entire available technologies list $\left[Y_{A T}^{\tau(\theta)}\right]$.

Any power system design variable of the new harmony, $\left[x^{\tau(\theta) n v}\right]=\left\{x_{11}^{\tau(\theta) n v}, x_{12}^{\tau(\theta) n v}, \ldots, x_{g k}^{\tau(\theta) n v}\right\}$, obtained by the memory consideration is examined to determine whether it is pitch-adjusted or not. Pitch adjustment is made by $\operatorname{PAR}^{\tau(\theta)}$. $\operatorname{PAR}^{\tau(\theta)}$ investigates better power system design in the neighboring of the current power system design. $\operatorname{PAR}^{\tau(\theta)}$ is applied as follows:

$\operatorname{PAD}^{\tau(\theta)}$ for $x_{g i}^{\tau(\theta) n v} \begin{cases}\text { Yes } & \text { If } \operatorname{ran}_{1}^{\tau(\theta)} \leq \operatorname{PAR}^{\tau(\theta)} \\ \text { No } & \text { If } \operatorname{ran}_{1}^{\tau(\theta)} \succ \operatorname{PAR}^{\tau(\theta)}\end{cases}$

$\left(\operatorname{ran}_{1}\right)^{\tau(\theta)}$ is the random number uniformly distributed over the interval $[0,1]$ and generated. If this random number is less than the $\operatorname{PAR}^{\tau(\theta)}, x_{g i}^{\tau(\theta) n v}$ is replaced with its neighbor technology components list. If this random number is not less than $\operatorname{PAR}^{\tau(\theta)}, x_{g i}^{\tau(\theta) n v}$ remains the same.

\subsubsection{Update the harmony memory}

If the new harmony $\left[x^{\tau(\theta) n v}\right]=\left\{x_{11}^{\tau(\theta) n v}, x_{12}^{\tau(\theta) n v}, \ldots\right.$, $\left.x_{g k}^{\tau(\theta) n v}\right\}$ is better than the worst power design in the $\operatorname{HM}^{\tau(\theta)}$, the new power design is included in the $\operatorname{HM}^{\tau(\theta)}$, and the existing worst harmony is excluded from the $\mathrm{HM}^{\tau(\theta)}$. In this process, it should be noted that $\mathrm{HM}^{\tau(\theta)}$ matrix is sorted again by unconstrained objective function and the same power design is not permitted in the $\mathrm{HM}^{\tau(\theta)}$ more than once.

\subsubsection{Stopping criterion}

The steps in Sects. 4.3.2 and 4.3.3 are repeated until a termination criterion is satisfied or reaching the number of improvisation (NI).

Calculate the optimal cost power design at the stage: $\tau(\theta) \rightarrow C(k)$ 
Table 1 Data of available different power components technologies

\begin{tabular}{|c|c|c|c|c|c|c|c|c|c|c|}
\hline \multirow[t]{2}{*}{ Subsystem \# } & \multirow[t]{2}{*}{ Data components \# $(\%)$} & \multicolumn{9}{|c|}{ Technologies \# } \\
\hline & & $\# 1$ & $\# 2$ & $\# 3$ & $\# 4$ & $\# 5$ & $\# 6$ & $\# 7$ & $\# 8$ & $\# 9$ \\
\hline \multirow[t]{3}{*}{ 1. Generators } & Reliability & 0.890 & 0.977 & 0.982 & 0.978 & 0.983 & 0.920 & 0.984 & l & l \\
\hline & Cost & 0.590 & 0.535 & 0.470 & 0.420 & 0.400 & 0.180 & 0.220 & l & l \\
\hline & Performance & 120 & 100 & 85 & 85 & 48 & 31 & 26 & I & l \\
\hline \multirow[t]{3}{*}{ 2. MT/HT transformers } & Reliability & 0.995 & 0.996 & 0.997 & 0.997 & 0.998 & l & l & l & l \\
\hline & Cost & 0.205 & 0.189 & 0.091 & 0.056 & 0.042 & l & l & l & l \\
\hline & Performance & 100 & 92 & 53 & 28 & 21 & l & l & l & l \\
\hline \multirow[t]{3}{*}{ 3. Lignes HT } & Reliability & 0.971 & 0.973 & 0.971 & 0.976 & l & l & l & l & l \\
\hline & Cost & 7.525 & 4.720 & 3.590 & 2.420 & l & l & l & l & l \\
\hline & Performance & 100 & 60 & 40 & 20 & l & l & l & l & l \\
\hline \multirow[t]{3}{*}{ 4. HT/MT transformers } & Reliability & 0.977 & 0.978 & 0.978 & 0.983 & 0.981 & 0.971 & 0.983 & 0.982 & 0.977 \\
\hline & Cost & 0.180 & 0.160 & 0.150 & 0.121 & 0.102 & 0.096 & 0.071 & 0.049 & 0.044 \\
\hline & Performance & 115 & 100 & 91 & 72 & 72 & 72 & 55 & 25 & 25 \\
\hline \multirow[t]{3}{*}{ 5. Lignes MT } & Reliability & 0.984 & 0.983 & 0.987 & 0.981 & l & l & l & l & l \\
\hline & Cost & 0.986 & 0.825 & 0.490 & 0.475 & I & I & I & I & / \\
\hline & Performance & 128 & 100 & 60 & 51 & l & l & l & l & / \\
\hline
\end{tabular}

Calculate the Availability of the optimal power design at the stage: $\tau(\theta) \rightarrow A(k)$.

\section{Illustrative example}

To illustrate the proposed harmony search algorithm, a numerical example is solved by use of the data given in Table 1. Each component of the subsystem is considered as a unit with total failures. Figure 6 contains the data of cumulative load demand. The maximum numbers of electrical components $k_{\max }$ in parallel are set to $(7,8,4,9,4)$. The numbers of music instruments are equal to the power subsystems. The simulation results depend greatly on the $\mathrm{HS}$ algorithm parameters values: $\mathrm{HMCR}=0.7$; $\mathrm{PAR}=0.5$; $\mathrm{HMS}=5$; and NI $=75$ for the first design. Several simulations are made for the expansion-planning power system where $0.7 \leq \mathrm{HMCR}^{\tau(1)} \leq 0.80 .5 \leq \operatorname{PAR}^{\tau(1)} \leq 0.65$; $\mathrm{HMS}^{\tau(1)}=0.5 ; \mathrm{HMS}^{\tau(\theta)}$ and NI ${ }^{\tau(1)}=100$ the best solution is obtained in 81 improvisations. The simulation was implemented to a real example taken from the Algerian network. Table 2 presents the obtained optimal electrical configuration.

\subsection{Description of the system to be optimized}

The electrical power station system which supplies the consumers is designed with five basic subsystems (stations) as depicted in Fig. 7. Figure 7 shows the detailed process of the electrical power station system distribution. The process of electrical power system distribution follows as: The elec-

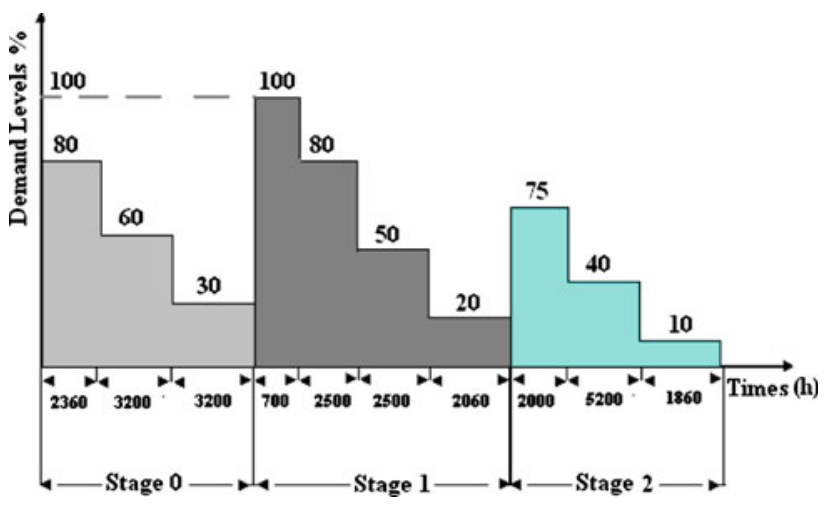

Fig. 6 Data of cumulative load-demand curves

trical power is generated from the station units (subsystem 1). Then transformed for high voltage (HT) by the HT transformers (subsystem 2) and carried by the HT lines mashed electrical network (subsystem 3). A second transformation in HT/MT transformers (subsystem 4) which supplies the MT load by the MT mashed electrical network (subsystem 5).

\subsubsection{Solution obtained by harmony search optimization algorithm}

See Table 2.

\subsubsection{Optimization result and discussion}

Much work in the field of reliability optimization analysis has been devoted using meta-heuristics methods. So, few of them treat the problem of expansion-planning EPP. The 
Table 2 Optimal solutions for expansion-planning problem EPP

\begin{tabular}{lllll}
\hline$A_{0}$ & Structure & $\begin{array}{l}\text { Stage 0: current year }(\text { cost = 13.60) } \\
A_{k}=0.9927\end{array}$ & $\begin{array}{l}\text { Stage 1: 2 years }(\text { cost }=17.703) \\
A_{k}=0.9935\end{array}$ & $\begin{array}{l}\text { Stage 2: 3 years }(\text { cost }=12.997) \\
A_{k}=0.997\end{array}$ \\
\hline 0.991 & Subsystem 1 & $4-4-6-7-4$ & $4-4-6-7-4 ; 1(4)^{*}-1(7)^{*}$ & $4-4-6-7-4 ; 1(4)^{* *}$ \\
& Subsystem 2 & $4-4-4-4-4-4-4$ & $4-4-4-4-4-4-4$ & $4-4-4-4-4-4 ; 2(4)^{* *}$ \\
& Subsystem 3 & $1-4$ & $1-4 ; 2(4)^{*}$ & $1-4$ \\
& Subsystem 4 & $7-7-7-9$ & $7-7-7-9 ; 1(8)^{*}$ & $7-7-7-9 ; 1(7)^{* *}$ \\
& Subsystem 5 & $4-4-4$ & $4-4-4 ; 2(4)^{*}$ & $4-4-4$ \\
\hline
\end{tabular}

Fig. 7 Synoptic of the detailed electrical network system

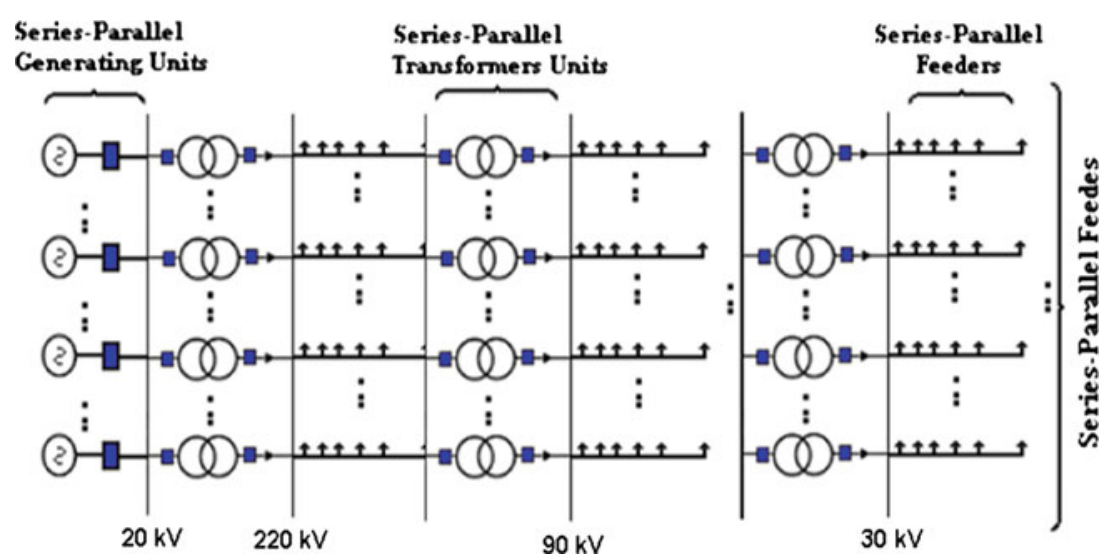

Fig. 8 Graph of different stage for EPP

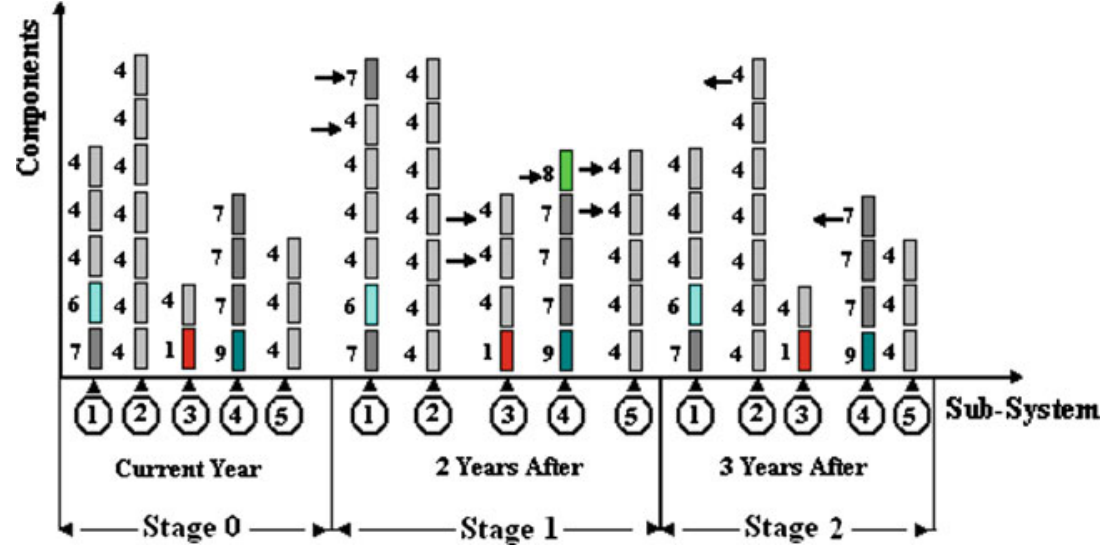

proposed harmony search method seeks system configuration that provides the optimal combination of components cost with adding or retrieving new component from the existing design structure under reliability constraint. Usually a gain in performance and reliability can only be obtained at an extra-trade-off between components (Fig. 8). To reinforce the existing design adding or retrieving components by replacement depend greatly on increasing or decreasing demand levels.

The natural objective function is to define the minimal investment design configuration under given reliability constraints at each stage. The whole of the results obtained by the proposed harmony search for given values of $A_{0}$ is illustrated in Table 2. The latter shows the best initial optimal power design and the optimal power design at each stage for the EPP for one desired reliability levels $A_{0}(0.991)$. Table 2 illustrates the computed cost and reliability index of the corresponding power design. The choice of these values affects strongly the solution. A quality solution measure is proposed taken from the NN method and the best solution is selected from the lower NN coefficient $\alpha=\frac{\text { Objective Function }(\%)}{\text { Constraint }(\%)}$. Since it is a meta- heuristic method only near optimal solutions can be obtained.

\section{Conclusion}

In this paper, we solve the electrical expansion-planning power design optimization which is a very interesting 
problem often reencountered in energy industry. It is formulated as redundancy optimization problem. The resolution of this problem uses a developing harmony search algorithm. This new algorithm for choosing an optimal series-parallel electrical power system design for EPP is proposed which minimizes total investment cost subject to reliability constraints. This algorithm seeks electrical components technologies according to their availability, nominal capacity (perfor-mance) and cost. Also defines the number and the kind of series-parallel electrical power components to put in each subsystem when consumers' demand changes by selects adding/or retrieving from initial power design. The proposed method allows a practical way to solve wide instances of reliability optimization problem of multistate systems without limitation on the diversity of electrical components technologies put in series-parallel. A combination is used in this algorithm based on the universal moment generating function UMGF and an HS algorithm.

Open Access This article is distributed under the terms of the Creative Commons Attribution Noncommercial License which permits any noncommercial use, distribution, and reproduction in any medium, provided the original author(s) and source are credited.

\section{References}

1. Levitin G, Lisnianski A, Ben-Haim H, Elmakis D (1997) Structure optimization of power system with different redundant elements. Electr Power Syst Res 43(1):19-27

2. Levitin G, Lisnianski A (2000) Optimal replacement scheduling in multi-state series-parallel systems. Int J Qual Reliab Eng 16:157162

3. Levitin G, Lianski A (1999) Optimal multistage modernization of power system subject to reliability and capacity requirement. Electr Power Syst Res 50:183-190

4. Ushakov IA, Levitin G, Lisnianski A (2002) Multi-state system reliability: from theory to practice. In: Proceedings of the 3rd international conference on mathematical methods in reliability, MMR. Trondheim, Norway, pp 635-638
5. Levitin G, Lisnianski A (2001) A new approach to solving problems of multi-state system reliability optimization. Int J Qual Reliab Eng 47(2):93-104

6. Chern MS (1992) On the computational complexity of reliability redundancy allocation in a series system. Oper Res Lett 11: 309-315

7. Tillman FA, Hwang L, Kuo W (1997) Optimization techniques for system reliability with redundancy - a review. IEEE Trans Reliab $26 \mathrm{~N}^{\circ}(3): 148-155$

8. Kuo W, Prasad VR (2000) An annotated overview of system-reliability optimization. IEEE Trans Reliab 49(2):176-187

9. Ushakov I (1997) Optimal standby problems and a universal generating function. Sov J Comput Syst Sci 25(N 4):79-82

10. Lisnianski A, Levitin G, Ben-Haim H, Elmakis D (1996) Power system structure optimization subject to reliability constraints. Electr Power Syst Res 39(2):145-152

11. Levitin G (2000) Multi-state series-parallel system expansionscheduling subject to availability constraints. IEEE Trans Reliab 49(1):71-79

12. Geem ZW (2006) Optimal cost design of water distribution networks using harmony search. Eng Optim 38(3):259-280

13. Zeblah A, Chatelet E, Samrout M, Yalaoui F, Massim Y (2008) Series-parallel power system optimization using harmony search algorithm. Int J Power Energy Convers (in press)

14. Lee KS, Geem ZW, Lee S-H, Bae K-W (2005) The harmony search heuristic algorithm for discrete structural optimization. Eng Optim 37(7):663-684

15. Ushakov I (1986) Universal generating function. Sov J Comput Syst Sci 24(5):118-129

16. Ross B (1993) Introduction to probability models. Academic Press, New York

17. Murchland JD (1975) Fundamental concepts and relations for reliability analysis of multi-state systems. In: Barlow R, Fussell J, Singpurwalla N (eds) Reliability and fault tree analysis. SIAM, Philadelphia

18. Levitin G, Lisnianski A, Ben-Haim H, Elmakis D (1998) Redundancy optimization for series-parallel multi-state systems. IEEE Trans Reliab 47(2): 165-172

19. Billinton R, Allan A (1990) Reliability evaluation of power systems. Pitman, New York

20. Omran MGH, Mahdavi M (2008) Global-best harmony search. Int J Appl Math Comput 198:643-656

21. Mahdavi M, Fesanghary M, Damangir E (2007) An improved harmony search algorithm for solving optimization problems. Int J Appl Math Comput 188:1567-1579 